\title{
Indicators of compliance for developmental follow-up of infants discharged from a regional NICU*
}

\author{
Vedika Nehra', Maria Pici², Paul Visintainer ${ }^{3}$ \\ and Jordan S. Kase ${ }^{2-4, * *}$ \\ 1 School of Medicine, New York Medical College, \\ Valhalla, NY, USA \\ 2 The Children's Rehabilitation Center, White Plains, \\ NY, USA \\ ${ }^{3}$ Department of Epidemiology, New York Medical \\ College, School of Public Health, Valhalla, NY, USA \\ ${ }^{4}$ The Division of Newborn Medicine, Department of \\ Pediatrics, Maria Fareri Children's Hospital at \\ Westchester Medical Center, Valhalla, NY, USA
}

discharge positively correlated with improved follow-up attendance. The severity of patient disease in the NICU did not impact follow-up rates. As a result close attention needs to be paid to factors which influence compliance with outpatient follow-up for developmental screening.

Keywords: Continuity of care after hospital discharge; developmental follow-up care; direct patient contact; neonatal intensive care unit (NICU); patient compliance; preterm infant.

\section{Introduction}

Over half a million infants are born prematurely each year in the US. The last two decades alone have demonstrated a $36 \%$ increase in preterm births with $\sim 12.8 \%$ of all infants now being born before 37 weeks of gestation [13]. This steady increase in preterm birth rates paired with reduced infant mortality due to advancements in antenatal and neonatal care has continually raised the number of surviving preterm infants $[1,6]$.

Preterm infants and infants born with high-risk medical conditions requiring admission to the neonatal intensive care unit (NICU) are at increased risk for developmental delay and atypical development [8-10, 20]. Previously, only very preterm, or low birth weight infants were the focus of neonatal developmental follow-up programs, however, it is now recognized that moderately preterm and late preterm infants also exhibit significant delays necessitating therapeutic treatment [11]. A recent study by Petrini et al. [15] demonstrated that decreasing gestational age was associated with increased incidence of cerebral palsy and developmental delay/mental retardation even for late preterm infants (34-36 weeks gestation). In fact, compared to full-term infants, late preterm infants were three times more likely to develop cerebral palsy. Chyi et al. [3] looked at moderately preterm (32-33 weeks gestation) and late preterm children at school age. They showed late-preterm infants were more likely to display below average reading competence from kindergarten through grade five, whereas moderately preterm infants were more likely to be enrolled in individual education and special education programs at the same grade levels.

It is well recognized that early detection and early intervention with therapeutic services assures the greatest 
potential outcome for at risk children by minimizing and sometimes even preventing future complications $[5,7$, 12, 14, 16-18]. Neonatal developmental follow-up programs are designed to specifically identify, assess, and track children who are susceptible to developmental delay secondary to morbidities encountered in the perinatal period. Comprehensive follow-up care involves providing regularly scheduled developmental assessments during which screening is conducted for sensory, fine and gross motor impairments, as well as speech and behavioral delays [4]. In conjunction with medical, psychological and social evaluation, these screenings create a holistic approach in evaluating the NICU graduate and optimizing their care.

The follow-up visit also provides an important opportunity for physicians to educate parents about potential health complications and address parental concerns regarding the specific developmental needs of these "at risk" children [2, 10, 14, 19]. Accordingly, lack of compliance with follow-up appointments poses a major obstacle to providing adequate developmental care.

Despite its potential benefits, compliance with developmental follow-up is often overlooked by parents as well as other health care professionals. Specific factors affecting compliance with follow-up care are yet unknown. Therefore, the objective of this study is to identify indicators which may predict the likelihood of compliance for patient visits at a regional neonatal follow-up program (RNFUP) after discharge from a NICU admission.

\section{Patients and methods}

This is a retrospective observational cohort analysis of former NICU patients at the regional NICU (RNICU) of Maria Fareri Children's Hospital at Westchester Medical Center. Subjects included for analysis were all patients who were discharged from RNICU and who were referred to the RNFUP between July $1^{\text {st }}$ 2006 and June $30^{\text {th }} 2007$.

\section{Patient contact}

In an attempt to improve neonatal follow-up compliance, beginning July $1^{\text {st }}$ of 2006 an attempt was made to contact patients' guardians by a neonatal fellow within 1 week of discharge from the RNICU. Fellows were to inquire about the status of the patient at home, assure that all outpatient appointments had been made, and answer any questions or concerns raised by the guardian at that time. Information from these telephone conversations was documented in a questionnaire format and utilized for analyses.

\section{Data collection}

Referred patients Discharge summaries for patients who were referred to the RNFUP were reviewed to obtain data regarding patient descriptives and morbidities in the perinatal period.

A total of 16 patient descriptives and 12 patient morbidities were analyzed. Patient descriptives included birth information, maternal factors, patient care needs/interventions, and patient transfer status (Table 1). Patient morbidities were those experienced in the newborn period including respiratory conditions, infectious, and congenital anomalies. These morbidities helped define the severity of illness of the neonate (Table 2).

Table 1 Descriptives for patients referred to the regional neonatal follow-up program (RNFUP).

\begin{tabular}{|c|c|c|}
\hline & $\begin{array}{l}\text { Attended follow-up } \\
\mathrm{n}=176\end{array}$ & $\begin{array}{l}\text { Did not attend follow-up } \\
n=122\end{array}$ \\
\hline \multicolumn{3}{|l|}{ Birth information } \\
\hline$<37$ weeks gestation, $\mathrm{n}(\%)$ & $145(60.9)$ & $93(39.1)$ \\
\hline Multiple gestation, n (\%) & $34(57.6)$ & $25(42.3)$ \\
\hline Cesarean section, $\mathrm{n}(\%)$ & $117(65.0)$ & $63(35.0)$ \\
\hline \multicolumn{3}{|l|}{ Maternal factors } \\
\hline Maternal age, mean $\pm \mathrm{SD}^{*}$ & $30.19 \pm 6.27$ & $27.83 \pm 6.78$ \\
\hline Maternal drug use, $\mathrm{n}(\%)^{\star \star}$ & $2(11.8)$ & $15(88.2)$ \\
\hline Maternal preeclampsia, $\mathrm{n}(\%)$ & $26(66.7)$ & $13(33.3)$ \\
\hline Non-English speaking mother, n (\%) & $28(75.7)$ & 9 (24.3) \\
\hline \multicolumn{3}{|l|}{ Patient care needs/interventions } \\
\hline Patient contacted post-discharge, $\mathrm{n}(\%)^{*}$ & $155(65.1)$ & $83(34.9)$ \\
\hline Early intervention referral, $\mathrm{n}(\%)^{\star}$ & $120(63.8)$ & $68(36.1)$ \\
\hline Number of in-hospital referrals, mean $\pm S D$ & $1.68 \pm 2.14$ & $1.75 \pm 1.95$ \\
\hline Number of appointments post-discharge, mean \pm SD & $3.34 \pm 1.47$ & $3.47 \pm 1.65$ \\
\hline Patient discharged home on oxygen, $\mathrm{n}(\%)$ & $9(56.3)$ & $7(43.7)$ \\
\hline Patient discharged with a home monitor, $\mathrm{n}(\%)$ & $25(65.8)$ & $16(32.7)$ \\
\hline Visiting nurse service post-discharge, n (\%) & $33(67.3)$ & $13(34.2)$ \\
\hline \multicolumn{3}{|l|}{ Patient transfer } \\
\hline Patient transferred into RNICU, n (\%) & $59(54.1)$ & $50(45.9)$ \\
\hline Patient transferred out of RNICU, $n(\%)^{\star *}$ & $3(10.7)$ & $25(89.3)$ \\
\hline
\end{tabular}

${ }^{*} \mathrm{P}<0.05 ;{ }^{*} \mathrm{P}<0.001$.

$\mathrm{RNICU}=$ regional neonatal intensive care unit. 
Table 2 Morbidities experienced in the newborn period for patients referred to regional neonatal follow-up program (RNFUP).

\begin{tabular}{lcc}
\hline & $\begin{array}{l}\text { Attended follow-up } \\
\mathrm{n}=176\end{array}$ & $\begin{array}{c}\text { Did not attend follow-up } \\
\mathrm{n}=122\end{array}$ \\
\hline Infection, $\mathrm{n}(\%)$ & $21(55.3)$ & $17(44.7)$ \\
Exchange transfusion, $\mathrm{n}(\%)$ & $4(100.0)$ & $0(0.0)$ \\
Congenital anomaly, $\mathrm{n}(\%)$ & $56(52.3)$ & $51(47.7)$ \\
Respiratory distress syndrome (RDS), $\mathrm{n}(\%)$ & $100(59.5)$ & $78(43.8)$ \\
Hyperbilirubinemia, $\mathrm{n}(\%)$ & $110(59.5)$ & $75(40.5)$ \\
Surgery in newborn period, $\mathrm{n}(\%)$ & $28(53.8)$ & $24(46.2)$ \\
Blood transfusion, $\mathrm{n}(\%)$ & $34(61.8)$ & $21(38.2)$ \\
Mechanical ventilation days, mean \pm SD & $3.84 \pm 10.9$ & $4.15 \pm 13.17$ \\
Continuous positive airway pressure (CPAP) days, mean \pm SD & $3.02 \pm 6.91$ & $2.57 \pm 6.45$ \\
Supplemental oxygen days, mean \pm SD & $3.34 \pm 8.06$ & $3.61 \pm 9.46$ \\
Total oxygen days, mean \pm SD & $9.78 \pm 19.41$ & $10.04 \pm 23.22$ \\
Length of stay days, mean \pm SD & $4.53 \pm 4.11$ & $4.23 \pm 4.31$
\end{tabular}

It is important to mention that causes for not contacting patients post-discharge included incorrect phone numbers and disconnected phone lines. Answering machines were utilized after failed attempts to contact caretakers directly and were categorized as patient contacted.

Non-referred patients In order to assess appropriate referral to the RNFUP, all patients discharged from the RNICU who were not referred for follow-up were evaluated for antenatal and neonatal morbidities which would put these children at greater risk for future developmental delays. This would identify those patients who should have been referred to the RNFUP but were not.

\section{Statistical analysis}

Patients who were referred to RNFUP were identified and dichotomized as compliant or non-compliant with follow-up appointments. Each patient descriptive and morbidity was then compared between those patients who were compliant and those who were not.

All patient data entries and subsequent analyses were performed utilizing the statistical software SPSS 11.5 (SPSS Inc, Chicago, IL, USA). The comparison of categorical variables between compliance groups was achieved with $\chi^{2}$ analysis, $t$ test compared the means of continuous variables, and MannWhitney $U$-rank sum test compared ordinal variables. Odds ratio calculated the benefit of post-discharge contact. Significance was defined as $\mathrm{P}<0.05$.

\section{Results}

A total of 436 patients were discharged from the RNICU between July $1^{\text {st }} 2006$ and June 30 2007 and were intended to be contacted by a neonatal fellow within 1 week of discharge (Figure 1).

Of the 436 patients to be contacted after discharge, $298(68.3 \%)$ patients were referred to the RNFUP. Of the referred patients, 238 (79.9\%) were contacted following discharge and 60 (20.1\%) were not contacted. A total of $176(59.1 \%)$ patients attended their follow-up appoint- ments at the RNFUP. Of those patients referred for follow-up and contacted by a fellow after discharge, 155 (65\%) were compliant. Whereas, compliance of those patients who were referred but not contacted postdischarge was 21 (35.0\%). Thus, patients who were both referred and contacted by a fellow within 1 week postdischarge from the RNICU were $85 \%$ more likely to attend their follow-up appointments at the RNFUP than patients who were referred but not contacted.

Maternal descriptives of significance were maternal age and maternal drug use (Table 1). Mothers who were slightly older, 30 years of age vs. 27 years of age, were more likely to attend follow-up (compliant: 30 years \pm 6.27 vs. non-compliant: 27 years \pm 6.78 ). On the other hand, maternal drug use significantly hindered compliance; mothers who used drugs during pregnancy were less likely to attend follow-up [compliant: 2 (11.8\%) vs. non-compliant: 15 (88\%)].

Early intervention referral upon discharge from the RNICU significantly affected compliance with follow-up at the RFNUP [(compliant: 120 (63.8\%) vs. non-compliant: 68 (36.1\%)] (Table 1).

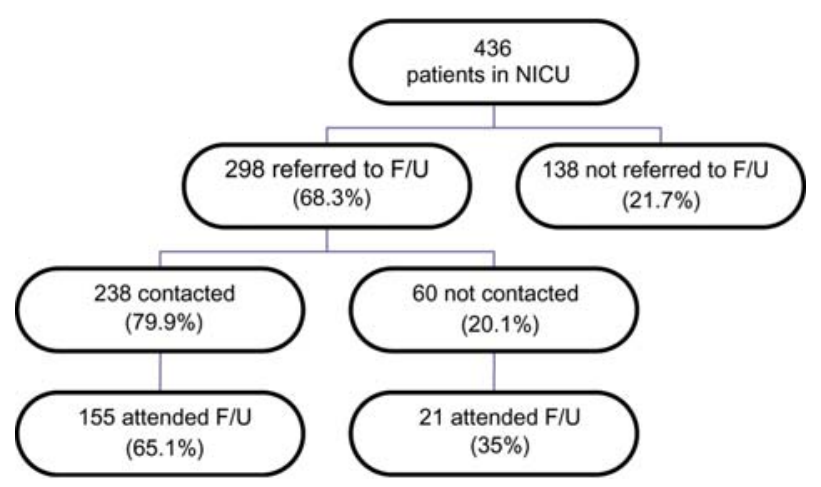

Figure 1 Patients intended for RNFUP referral and postdischarge contact.

$\mathrm{F} / \mathrm{U}=$ follow-up. 
Patient transfer status had a remarkable impact upon follow-up compliance. Patients who were transferred to outside NICUs were less likely to attend follow-up than those who were discharged directly from the RNICU [(transferred out: compliant: 3 (10.3\%), non-compliant 25 (89.3\%); no transfer: compliant: 79 (62.2\%), non-compliant: 48 (37.8\%)] (Table 1).

Of note, none of the newborn morbidities or gestational age at birth significantly affected patient compliance rates with follow-up (Tables 1 and 2 ).

One hundred and thirty-eight (31.7\%) patients were not referred to the follow-up program. Of those, 38 patients were preterm and 21 required surgery in the newborn period, both of which are high-risk conditions requiring neonatal developmental follow-up care.

\section{Discussion}

It is imperative that patients at risk for developmental delay are identified and referred for developmental follow-up prior to discharge. It is also important that actions are taken to ensure these children receive appropriate outpatient care to help them achieve their developmental potential. Our study sheds light on predictors of compliance and non-compliance with developmental follow-up care for patients discharged from a RNICU.

Addressing a child's developmental needs early in life is important for several reasons; it increases a caretakers awareness of his/her child's needs, it provides caretakers a means of educating themselves in regard to their child's medical conditions and developmental outcome, and it caries the promise of improving quality of life for a child and their family. All of which increase the likelihood that a child will meet his/her developmental potential later in life.

The first and perhaps most important step in assuring close follow-up care after RNICU discharge is the identification and subsequent referral of patients at increased risk for developmental delay to a RNFUP. Patient identification involves a thoughtful and holistic evaluation of all NICU graduates prior to discharge. An unexpected finding of this study is the number of patients who appeared to meet the criteria for developmental follow-up but were not referred. This suggests a need for improvement in identifying patients while they are still in the RNICU. The specific reasons for why these patients were not referred remains unclear and warrants further investigation.

We have identified four important predictors of patient compliance with follow-up as well as one very effective method for improving compliance. Infants of younger mothers, infants of mothers who use drugs during pregnancy, and infants who are transferred from the RNICU to a second outside hospital were less likely to be compliant with follow-up appointments at our RNFUP. Thus, these children will require the greatest efforts to assure follow-up attendance. On the other hand, direct referral for outpatient assessment by early intervention in conjunction with referral to the RNFUP appears to heighten a caregiver's awareness of the need for developmental follow-up and increases the likelihood of patient compliance.

Perhaps most strikingly, our study demonstrates that a single phone call made within 1 week of patient discharge in combination with developmental follow-up referral significantly increases the rate of compliance with developmental follow-up care. NICU graduates often have multiple outpatient appointments and their caretakers, already overwhelmed by the NICU admission, are often unable to recognize the importance of attending each scheduled appointment. A phone call later in the week not only helps remind caretakers of their child's appointments, but it also allows health professionals to educate the patient's family and reinforce the need for patient compliance. Additionally, during the telephone conversation caretakers are encouraged to voice their personal concerns and ask questions, in turn placing the caretaker at greater ease and better equipped to care for his/her child.

Although the specific number of scheduled outpatient appointments post-discharge did not impact attendance at RNFUP, we did not assess patient compliance with the other outpatient appointments. As previously identified, what is certain is that a phone call improves developmental follow-up compliance. Thus, it is possible that by reinforcing all appointment dates, that same phone call may have also improved compliance with all other outpatient medical services.

The scarcity of information in the literature regarding compliance with developmental follow-up necessitates further study. An important limitation of our study was that it only looked at antenatal, neonatal, preterm status and patient transfer status as they could be gathered from discharge summaries. Sociodemographic factors likely play an important role in patient compliance but were not assessed as they would require patient caretaker contact which was outside the realm of our study. A future investigation addressing sociodemographic factors would certainly provide valuable insight.

\section{Conclusion}

The analysis of our cohort of patients at the RNICU of Maria Fareri Children's Hospital has allowed us to identify several indicators of patient compliance with developmental follow-up. However, it is plausible that geographical and population variations may result in the identification of alternative predictors of compliance. Thus, while keeping our indicators in mind we recommend each RNICU analyze its specific patient cohort to best determine causes of compliance and non-compli- 
ance. We have also established a positive correlation with direct patient contact within a week after discharge and increased attendance at developmental follow-up appointments. We highly recommend the application of this simple technique to each discharge procedure to improve patient compliance with developmental follow-up appointments as well as all other follow-up appointments.

\section{Acknowledgements}

We would like to thank Mary Jackson for all her help and support with data collection for this study.

\section{References}

[1] Aylward GP. Neurodevelopmental outcomes of infants born prematurely. J Dev Behav Pediatr. 2005;26:427-40.

[2] Berger SP, Holt-Turner I, Cupoli JM, Mass M, Hageman JR. Neonatology update. Caring for the graduate from the neonatal intensive care unit. At home, in the office, and in the community. Pediatr Clin N Am. 1993;45:701-12.

[3] Chyi L, Lee H, Hintz S, Gould J, Sutcliffe T. School outcomes of late preterm infants: special needs and challenges for infants born at 32 to 36 weeks gestation. J Pediatr. 2008;153:25-31.

[4] Committee on Children and Disabilities, American Academy of Pediatrics. Developmental surveillance and screening for infants and young children. Pediatrics. 2001;108: 192-6.

[5] David Olds. Progress in improving the development of low birth weight newborns. Pediatrics. 2006;117:940-1.

[6] Fanaroff AA, Hack M, Walsh MC. The NICHD Neonatal Research Network: changes in practice and outcome during the first fifteen years. Semin Perinatol. 2003;27:281-7.

[7] Guralnick MJ. Effectiveness of early intervention for vulnerable children: a developmental perspective. Am J Ment Retard. 1998:102:319-45.

[8] Hack M, Fanaroff AA. Outcomes of children of extremely low birthweight and gestational age in the 1990's. Early Hum Dev. 1999;53:193-218.

[9] Harper RG, Rehman KU, Sia C, Buckwald S, Spinazzola $\mathrm{R}$, Schlessel J, et al. Neonatal outcome of infants born at 500 to 800 grams from 1990 through 1998 in a tertiary care center. J Perinatol. 2002;22:555-62.
[10] Hussey-Gardner B, McNinch A, Anastasi JM, Miller M. Early intervention best practice: collaboration among an $\mathrm{NICU}$, an early intervention program and an NICU followup program. Neonatal Network. 2002;21:15-22.

[11] Kalia J, Brumberg $H$, Visintainer P, Pici M, Kase, J. The comparison of enrollment in interventional therapies between late preterm (34/7-36/7 weeks gestation) and very preterm ( $<32$ weeks gestation) infants at 12 months corrected age. Pediatrics. 2009;123:804-9.

[12] Kaminer R, Jedrysek E. Early identification of developmental disabilities. Pediatric Annals. 1982;11:427-37.

[13] Martin JA, Hamilton BE, Sutton PD, Ventura SJ, Menacker F, Kirmeyer S, et al. Births: final data for 2006. Natl Vital Stat Rep. 2009;56:2.

[14] McCormick MC, Brooks-Gunn J, Buka SL, Goldman J, Yu $\mathrm{J}$, Salganik M, et al. Early intervention in low birth weight premature infants: results at 18 years of age for the infant health and development program. Pediatrics. 2006;117: 771-80.

[15] Petrini JR, Dias T, McCormick MC, Massolo ML, Green NS, Escobar GJ. Increased risk of adverse neurological development for late preterm infants. J Pediatr. 2009;154: 169-76.

[16] Reynolds AJ, Temple JA, Robertson DL, Mann EA. Longterm effects of an early childhood intervention on educational achievement and juvenile arrest a 15-year follow-up of low-income children in public schools. J Am Med Assoc. 2001;285:2339-46.

[17] Salokorpti T, Rautio T, Kajantie E, Von Wendt L. Is early occupational therapy in extremely preterm infants of benefit in the long run? Pediatr Rehabil. 2002;5:91-8.

[18] Salokorpti T, Sajaniemi N, Rajantie I, Hallback H, Hamalainen, Rita $\mathrm{H}$, et al. Neurodevelopment until the adjusted age of 2 years in extremely low birth weight infants after early intervention - a case control study. Pediatr Rehabil. 1998;2:157-63.

[19] Vohr BR, O'Shea M, Wright LL. Longitudinal multicenter follow-up of high-risk infants: why, who, when, and what to assess. Semin Perinatol. 2003;27:333-42.

[20] Wood NS, Marlow N, Costeloe K, Gibson AT, Wilkinson ARB. Neurologic and developmental disability after extremely preterm birth. New Engl J Med. 2000;343:37884.

The authors stated that there are no conflicts of interest regarding the publication of this article.

Received April 21, 2009. Accepted June 8, 2009. Previously published online August 13, 2009. 